\title{
A new multi-objective Jaya algorithm for optimization of modern machining processes
}

\author{
Rao, R.V. ${ }^{a,}{ }^{*}$, Rai, D.P. ${ }^{\text {, }}$ Ramkumar, J. ${ }^{\text {, }}$, Balic, J. ${ }^{c}$ \\ ${ }^{a}$ Department of Mechanical Engineering, Sardar Vallabhbhai National Institute of Technology, Surat, India \\ ${ }^{b}$ Department of Mechanical Engineering, Indian Institute of Technology, Kanpur, India \\ 'Production Engineering Institute, Faculty of Mechanical Engineering, University of Maribor, Slovenia
}

\begin{abstract}
A B S T R A C T
In this work, the multi-objective optimization aspects of plasma arc machining (PAM), electro-discharge machining (EDM), and micro electro-discharge machining $(\mu$-EDM) processes are considered. Experiments are performed and actual experimental data is used to develop regression models for the considered machining processes. A posteriori version of Jaya algorithm (MOJaya algorithm) is proposed to solve the multi-objective optimization models in a single simulation run. The PAM, EDM and $\mu$-EDM processes are optimized using MO-Jaya algorithm and a set of Pareto-efficient solutions is obtained for each of the considered machining processes and the same is reported in this work. This Pareto optimal set of solutions will provide flexibility to the process planner to choose the best setting of parameters depending on the application. The aim of this work is to demonstrate the performance of MO-Jaya algorithm and to show its effectiveness in solving the multi-objective optimization problems of machining processes.
\end{abstract}

(c) 2016 PEI, University of Maribor. All rights reserved.

\section{ARTICLE INFO}

Keywords:

Plasma arc machining

Electro-discharge machining

Micro-electro-discharge machining

Multi-objective optimization

Jaya algorithm

Posteriori approach

Sustainability

*Corresponding author:

ravipudirao@gmail.com

(Rao, R.V.)

Article history:

Received 10 November 2016

Revised 25 November 2016

Accepted 1 December 2016

\section{References}

[1] De Wolf, D., Cardon, L., Balic, J. (2010). Parameters effecting the quality of the electrical discharge machining process, Advances in Production Engineering and Management, Vol. 5, No. 4, 245-252.

[2] Aich, U., Banerjee, S. (2016). Application of teaching learning based optimization procedure for the development of SVM learned EDM process and its pseudo Pareto optimization, Applied Soft Computing, Vol. 39, 64-83, doi: 10.1016/i.asoc.2015.11.002.

[3] Zhang, T.Y., Owodunni, O., Gao, J. (2015). Scenarios in multi-objective optimization of process parameters for sustainable machining, Procedia CIRP, Vol. 26, 373-378, doi: 10.1016/i.procir.2014.07.186.

[4] Gupta, M.K., Sood, P.K., Sharma, V.S. (2016). Optimization of machining parameters and cutting fluids during nano-fluid based minimum quantity lubrication turning of titanium alloy by using evolutionary techniques, Journal of Cleaner Production, Vol. 135, 1276-1288, doi: 10.1016/i.jclepro.2016.06.184.

[5] Chandrasekaran, M., Muralidhar, M., Krishna, C.M., Dixit, U.S. (2010). Application of soft computing techniques in machining performance prediction and optimization : A literature review, The International Journal of Advanced Manufacturing Technology, Vol. 46, No. 5, 445-464, doi: 10.1007/s00170-009-2104-x.

[6] Jiang, Z., Zhou, F., Zhang, H, Wang, Y., Sutherland, J.W. (2015). Optimization of machining parameters considering minimum cutting fluid consumption, Journal of Cleaner Production, Vol. 108 (part A), 183-191, doi: 10.1016/ j.jclepro.2015.06.007.

[7] Yusup, N., Zain, A.M., Hashim, S.Z.M. (2012). Evolutionary techniques in optimizing machining parameters: Review and recent applications (2007-2011), Expert Systems with Applications, Vol. 39, No. 10, 9909-9927, doi: 10.1016/j.eswa. 2012.02.109. 
[8] Rao, R.V., Kalyankar, V.D. (2014) Optimization of modern machining processes using advanced optimization techniques: A review, The International Journal of Advanced Manufacturing Technology, Vol. 73, No. 5, 11591188, doi: 10.1007/s00170-014-5894-4.

[9] Goswami, D., Chakraborty, S. (2015), Parametric optimization of ultrasonic machining process using gravitational search and fireworks algorithms, Ain Shams Engineering Journal, Vol. 6, No. 1, 315-331, doi: 10.1016/ j.asej.2014.10.009.

[10] Saha, A., Mondal, S.C. (2016). Multi-objective optimization in WEDM process of nanostructured hardfacing materials through hybrid techniques, Measurement, Vol. 94, 46-59, doi: 10.1016/i.measurement.2016.07.087.

[11] Maity, K.P., Bagal, D.K. (2015). Effect of process parameters on cut quality of stainless steel of plasma arc cutting using hybrid approach, The International Journal of Advanced Manufacturing Technology, Vol. 78, No. 1, 161-175, doi: $10.1007 / \mathrm{s} 00170-014-6552-6$.

[12] Rao, R.V., Rai, D.P., Balic, J. (2016). Multi-objective optimization of machining and micro-machining processes using non-dominated sorting teaching-learning-based optimization algorithm, Journal of Intelligent Manufacturing, doi:10.1007/s10845-016-1210-5.

[13] Li, C., Xiao, Q., Tang, Y., Li, L. (2016). A method integrating Taguchi, RSM and MOPSO to CNC machining parameters optimization for energy saving, Journal of Cleaner Production, Vol. 35, 263-275, doi: 10.1016/i.jclepro. 2016.06 .097$.

[14] Rao, R.V. (2016). Jaya: A simple and new optimization algorithm for solving constrained and unconstrained optimization problems, International Journal of Industrial Engineering Computations, Vol. 7, No. 1, 19-34, doi: 10.5267/j.ijiec.2015.8.004. 


\section{APEM}

\title{
Nov večkriterijski Jaya algoritem za optimizacijo sodobnih obdelovalnih postopkov
}

\author{
Rao, R.V. ${ }^{a,}{ }^{*}$, Rai, D.P. ${ }^{a}$, Ramkumar, J. $^{\text {, }}$, Balic, J. ${ }^{c}$ \\ a Department of Mechanical Engineering, Sardar Vallabhbhai National Institute of Technology, Surat, India \\ ${ }^{b}$ Department of Mechanical Engineering, Indian Institute of Technology, Kanpur, India \\ 'Production Engineering Institute, Faculty of Mechanical Engineering, University of Maribor, Slovenia
}

\begin{abstract}
POVZETEK
$\mathrm{V}$ tem delu smo izvedli večkriterijsko optimizacijo obdelave s plazmo (angl. PAM), elektroerozijske obdelave (angl. EDM) in mikroelektroerozijske obdelave (angl. $\mu$-EDM). Izvedli smo niz eksperimentov, dobljene podatke pa uporabili za razvoj regresijskih modelov za proučevane obdelovalne postopke. $\mathrm{Za}$ večkriterijko optimizacijo zgoraj omenjenih obdelovalnih postopkov smo predlagali posteriorno različico algoritma Jaya (MO-Jaya algoritem). Za vsak obdelovalni postopek smo dobili niz Pareto optimalnih rešitev, ki omogočajo veliko fleksibilnost, saj lahko načrtovalec proizvodnega postopka izbere najboljši niz obdelovalnih parametrov z ozirom na vrsto aplikacije (obdelave), ki jo mora izvesti. Namen te raziskave je bil prikazati zmogljivost algoritma MOJaya in njegovo učinkovitost pri reševanju večkriterijskih optimizacijskih problemov pri sodobnih obdelovalnih postopkih.
\end{abstract}

(C) 2016 PEI, University of Maribor. All rights reserved.

\section{PODATKI O ČLANKU}

Ključne besede:

Obdelava s plazmo

Elektro erozijska obdelava

Mikro elektro erozijska obdelava

Večkriterijska optimizacija

Jaya algoritem

Posteriorni pristop

Trajnost

*Kontaktna oseba:

ravipudirao@gmail.com

(Rao, R.V.)

Zgodovina članka:

Prejet 10. novembra 2016

Popravljen 25. novembra 2016

Sprejet 1. decembra 2016 